\title{
Exigências nutricionais para bagres nativos do Brasil: uma revisão
}

\author{
Nutritional requirements for native Brazilian catfish: a review \\ Requisitos nutricionales para el bagre nativo Brasileño: una revisión
}

\section{Resumo}

A piscicultura brasileira se encontra em constante crescimento, sendo que $34,7 \%$ dessa produção é proveniente de espécies nativas. Dentre as espécies nativas se destacam os bagres, peixes de couro que não apresentam espinhos intramusculares e são bem aceitos pelo mercado consumidor. A presente revisão aborda a relação entre produção e pesquisa dos principais bagres nativos do Brasil (Pseudoplatystoma e Rhamdia quelen) e traz um panorama sobre os principais estudos ligados às exigências nutricionais destas espécies. Tem ainda a finalidade de embasar novas pesquisas e fornecer um compilado de trabalhos que podem auxiliar na formulação de dietas específicas para estas espécies. Foram identificados quatro pontos relevantes nesta revisão: (i) a produção de surubim é basicamente focada em peixe híbridos, enquanto que para as pesquisas há uma variedade muito grande nas espécies utilizadas; (ii) é necessária uma adequada padronização das dietas em termos de balanço nutricional e aspectos de fabricação como extrusão ou peletização; (iii) são escassas as dietas comerciais específicas para espécies nativas, especialmente em se tratando de micronutrientes, possivelmente impactando a indústria com poucas opções comerciais e linha de produtos; (iv) discrepância entre as pesquisas científicas com nutrição e o volume de produção comercial dessas espécies. A produção de peixes com limitado conhecimento nutritional é possível, porém requer um esforço comum entre a academia e a cadeia produtiva. O desenvolvimento de uma estratégia científica na área de nutrição alinhada com os interesses da indústria é indispensável para a expansão da atividade.

Palavras-chave: Nutrição; Produção; Pseudoplatystoma; Rhamdia quelen.

\begin{abstract}
Brazilian fish farming is in expansion, with native species corresponding to $34.7 \%$ of its production. Catfish is one of the main farmed native species without intramuscular spines and are well accepted by the consumers. This review addresses the relationship between production and research of the main native catfish species in Brazil (Pseudoplatystoma and Rhamdia quelen) and also provides an overview of the main nutritional requirements studies with these species. It also aims to provide novel insights on nutrition research and species-specific formulations. Four main points were identified in this review: (i) surubim production is basically focused on hybrid fish, while many species have been investigated; (ii) benchmark diets in terms of nutritional composition and manufacturing technology such as extrusion or pelletization is necessary; (iii) tailored commercial diets for native species are scarce, especially when it comes to micronutrients, possibly impacting the industry with few commercial options and product lines; (iv) discrepancy between nutrition scientific research and the volume of commercial production of these
\end{abstract}


species. Farming fish species with limited nutritional knowledge is possible, but it requires a common effort between the academy and the supply chain. The development of a scientific strategy in the area of nutrition aligned with the interests of the industry is essential for the expansion of the activity.

Keywords: Nutrition; Production; Pseudoplatystoma; Rhamdia quelen.

\section{Resumen}

La piscicultura brasileña está en constante crecimiento, con un 34,7\% de esta producción proveniente de especies nativas. Entre las especies autóctonas destacan el bagre, pescado de cuero que no tienen espinas intramusculares y son bien aceptados por el mercado consumidor. Esta revisión aborda la relación entre producción e investigación de los principales bagres nativos de Brasil (Pseudoplatystoma y Rhamdia quelen) y proporciona una visión general de los principales estudios vinculados a los requerimientos nutricionales de estas especies. También tiene el propósito de apoyar nuevas investigaciones y proporcionar una recopilación de trabajos que puedan ayudar en la formulación de dietas específicas para estas especies. En esta revisión se identificaron cuatro puntos relevantes: (i) la producción de surubim se centra básicamente en peces híbridos, mientras que para la investigación existe una gran variedad en las especies utilizadas; (ii) es necesaria una adecuada estandarización de las dietas en términos de equilibrio nutricional y aspectos de fabricación como extrusión o peletización; (iii) las dietas comerciales específicas para especies nativas son escasas, especialmente cuando se trata de micronutrientes, posiblemente impactando la industria con pocas opciones comerciales y línea de productos; (iv) discrepancia entre la investigación científica sobre nutrición y el volumen de producción comercial de estas especies. La producción de pescado con conocimientos nutricionales limitados es posible, pero requiere un esfuerzo común entre la academia y la cadena de producción. El desarrollo de una estrategia científica en el área de la nutrición alineada con los intereses de la industria es fundamental para la expansión de la actividad.

Palabras clave: Nutrición; Producción; Pseudoplatystoma; Rhamdia quelen.

\section{Introdução}

No Brasil, a produção aquícola cresceu significativamente nos últimos anos (Peixe BR, 2021). Embora o grande avanço da aquicultura nacional seja oriundo da produção de tilápias, os peixes nativos representam uma boa fatia do mercado (Peixe BR, 2021). Em 2020, a produção de peixes nativos apresentou 34,7\% do total de peixes de água doce produzido, totalizando 287.930 toneladas, mesmo com uma queda de 3,2\% comparado com o ano anterior. Essa queda na produção, pode estar diretamente relacionada com o alto custo de produção, falta de investimentos nos principais estados produtores, regras ambientais, dificuldade de logística ou ainda problemas de comercialização, devido a um processamento adequado dos peixes (Peixe BR, 2021). Vale ressaltar que todos os estados brasileiros, exceto o Ceará, são responsáveis por alguma parcela da produção de peixes nativos (Peixe BR, 2021).

Dentre os problemas citados anteriormente a nutrição adequada dos peixes pode estar diretamente relacionada aos custos de produção, uma vez que, a alimentação desses animais representa a maior porcentagem dos gastos produtivos, cerca de $70 \%$ dos custos operacionais (Cyrino et al., 2010). Deste modo, a formulação das dietas de cada espécie deve levar em conta diversos fatores, como as exigências nutricionais, o hábito alimentar, o sistema de criação, a digestibilidade dos ingredientes, entre outros (Hemre et al., 2002).

O emprego de dietas formuladas para peixes exóticos, tais como, a truta arco-íris (Oncorhynchus mykiss), bagre do canal (Ictalurus punctatus), tilápia-do-Nilo (Oreochromis niloticus), carpa (Ciprinus carpio) e o salmão do Atlântico (Salmo salar) é comumente utilizada na nutrição de peixes nativos. Com isso, a utilização de dietas desbalanceadas e inaptas é comum, podendo comprometer a saúde (escassez ou excesso de nutrientes) e/ou o desempenho dos animais. Além disso, expressivo impacto ambiental advindo do não aproveitamento das dietas também pode ser observado (NRC, 2011).

No que diz respeito aos estudos científicos com nutrição de organismos aquáticas, a realidade é o uso de dietas experimentais na forma peletizada. Na indústria, por sua vez, dietas extrusadas são formuladas e comercializadas ao produtor (Moro, 2015). Adicionalmente, a falta de recursos para pesquisas ou a falta de comunicação entre ambas as interessadas (comunidade acadêmica e cadeia produtiva), defasam ainda mais as informações existentes. Essa ausência de padronização, além de também aumentar os custos produtivos, podem mascarar o máximo potencial genético e produtivo dos animais (NRC, 
2011; Moro, 2015). Ainda neste sentido, a inexistência de uma base de dados nutricionais para espécies nativas dificulta ainda mais a ascensão da atividade, bem como, a eficiência da cadeia produtiva (NRC, 2011). Se considerarmos ainda, os mais variados sistemas de produção e cultivo existentes, todos os entraves citados anteriormente ganham mais destaque. Dessa forma, estudos sobre as exigências nutricionais das espécies nativas são indispensáveis e necessários para alavancar e desenvolver a atividade aquícola nacional.

Dentre as mais variadas espécies nativas existentes, podem ser destacados os bagres, nome comum dado aos peixes da ordem Siluriformes. Nessa ordem, são conhecidos em torno de 2.200 espécies que podem ser divididas em 40 famílias. Estes peixes podem ser encontrados em todo o mundo exceto na Antártida, no entanto, mais da metade das espécies são nativas da América do Sul. Estas espécies possuem características peculiares, tais como a presença de barbilhões, com função sensorial e de localização. São peixes desprovidos de escamas, também chamados peixes de couro. Por não apresentarem espinhos intramusculares, sua aceitação pelo mercado consumidor é favorecida. No Brasil, destacam-se entre os bagres, os surubins do gênero Pseudoplatystoma com maior renome na região Centro Oeste (Valenti et al., 2021) e o jundiá (Rhamdia quelen) com maior produção na região sul, devido sua resistência às baixas temperaturas (Baldisserotto \& Gomes, 2013).

Por essas inúmeras vantagens, se faz necessário conhecer o atual momento da nutrição destes bagres nativos do Brasil. Através do adequado levantamento de características e de estudos já realizados das espécies, é possível pontuar as principais lacunas e estimular novos e importantes estudos sobre o tema. Adicionalmente, esse levantamento possibilitará o desenvolvimento de dietas comerciais específicas, que certamente são o "ponto-chave" para alavancar a produção e viabilidade econômica destas espécies. Diante disso, o objetivo da presente revisão foi realizar um levantamento dos principais estudos sobre a nutrição de bagres nativos brasileiros (surubins - pintado e cachara - e jundiá) e refletir sobre o conhecimento existente e sua real aplicação no setor produtivo.

\subsection{Pseudoplatystoma}

O gênero Pseudoplatystoma compreende os maiores peixes da família Pimelodidae, que são encontrados nas principais bacias hidrográficas Sul-americanas como a do São Francisco, Prata e a Amazônica (Baldisserotto \& Gomes, 2013). Algumas espécies são conhecidas popularmente como surubins e são criadas comercialmente como o surubim-pintado $(P$. coruscans) e o surubim-cachara ( $P$. reticulatum), além dos híbridos resultantes do cruzamento destas espécies, sendo o principal o Pintachara. Ele é originado através do cruzamento da femea de Pseudoplatystoma corruscans com o macho do Pseudoplatystoma reticulatum (Gonçalves, 2014). Filho et al. (2001) mostraram que o arranjo intestinal do surubim é compatível com a maioria dos peixes carnívoros. Uma vez que, seu intestino é quase retilíneo, apresentando algumas alças no final da sua porção média, que possivelmente seja uma adaptação a um regime de onivoria.

Esses peixes possuem ainda, excelente aceitação no mercado por apresentar carne saborosa, baixo teor de gordura e ausência de espinhos intramusculares, reconhecidos como um produto nobre (Crepaldi et al., 2007). Em escala comercial, por exemplo, o cachara é estocado com peso de $15 \mathrm{~g}$ em tanques de terra após treinamento alimentar para aceitação de ração. Sua despesca ocorre quando ele atinge aproximadamente $2 \mathrm{~kg}$, o que normalmente ocorre após 11 a 13 meses de cultivo (Cunha et al., 2015). Em 2018, a produção nacional dessa espécie foi de 11.505 toneladas, gerando um montante de $\mathrm{R} \$ 121$ milhões (IBGE, 2019).

\subsection{Rhamdia quelen}

Alguns períodos anuais de baixas temperaturas dificultam a criação das espécies de peixes tradicionais como a tilápia, principalmente na região sul do Brasil (Fracalossi et al., 2004). Isto pode ser visto como um entrave na produção, ou ainda, como uma oportunidade para produzir um produto diferenciado. Entre as espécies destaca-se o jundiá, pertencente à seguinte 
divisão taxonômica: classe: Osteichthyes, série: Teleostei, ordem: Siluriformes, família: Pimelodidae, gênero: Rhamdia, espécie: Rhamdia quelen.

Espécie nativa com potencial para a aquicultura brasileira, principalmente por tolerar temperaturas amenas. Apresenta ainda, crescimento rápido, carne sem espinhos intramuscular e bom rendimento de carcaça (Melo et al., 2002). Além disso, por apresentar fácil reprodução e aceitação aos mais variados tipos de alimentos, torna-se uma espécie com potencial para o cultivo (Parra et al., 2008). O comportamento do jundiá sugere uma forma de vida bentônica, sendo um predador oportunista (VillaresJunior \& Goitein, 2015). Seu hábito alimentar é muito variado, considerado de amplo espectro, alimentando-se de insetos terrestres e aquáticos, crustáceos e peixes, além de aceitar muito bem rações (Broggi et al., 2017). Estes animais possuem a estrutura bucal de tamanho grande, sem dentes e com três pares de barbilhões sensitivos do lado externo (Junior et al., 2011).

\section{Produção X Pesquisa}

Segundo o IBGE (2019), os peixes nativos mais produzidos no país no ano de 2018 foram os peixes redondos com $85 \%$ da produção. Dentre eles, o tambaqui, pacu, pirapitinga e seus híbridos se destacam. Na sequência, os surubins do gênero Pseudoplatystoma contribuem com 6,22\%, sendo estes, o pintado, o cachara e os seus híbridos. Peixes do gênero Brycon, como o matrinxã e a piracanjuba são os próximos com 3,84\% da produção e o pirarucu com 1\%. Outras espécies que compõe os 4,07\% da produção de peixes nativos são: jundiá, piau, piava, curimatã, traíra, lambari, tucunaré, dourado, entre outros (Figura 1).

Figura 1. Produção de peixes no Brasil no ano de 2018 com ênfase nos principais peixes nativos.

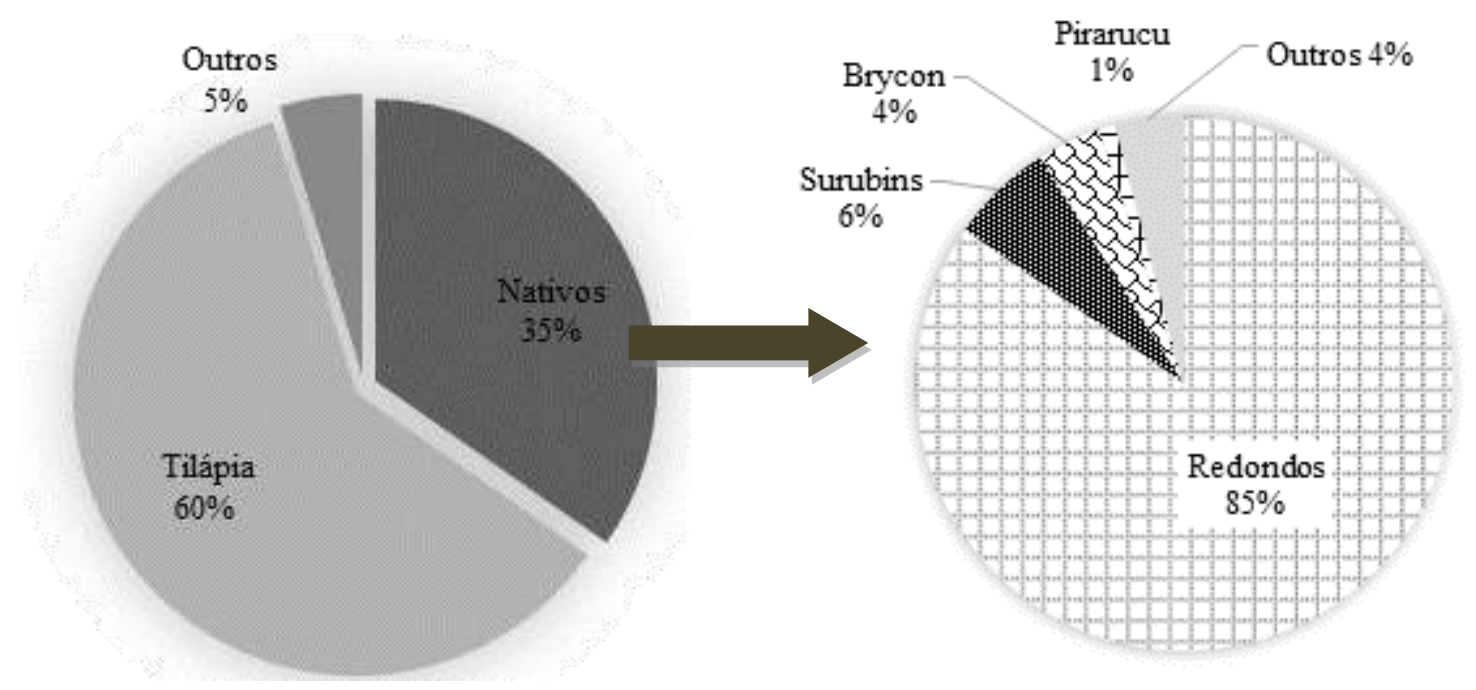

Fonte: Autores.

Realizou-se uma pesquisa na base de dados científica Science Direct, com o intuito de levantar o número de publicações (pesquisas científicas) sobre as espécies nativas, realizadas até 2020. Para tal, utilizou-se nessa busca as palavraschave "nutrition + nome científico da espécie". Como resultado, encontrou-se um aumento expressivo no número de trabalhos científicos nos últimos anos para ambas as espécies (Figura 2). Dentre esses trabalhos, há um destaque para o volume de trabalhos desenvolvidos com jundiá, no entanto, esses valores ainda são pequenos quando comparados, por exemplo, com trabalhos desenvolvidos com tilápias (1.543 trabalhos somente entre os anos de 2015 e 2020). Vale ressaltar que a tilápia tem uma produção global e as pesquisas com esta espécie são realizadas no mundo todo. 
Figura 2. Resultado da busca por "nutrition + nome científico" no ScienceDirect, relacionados de 2000 a 2020.

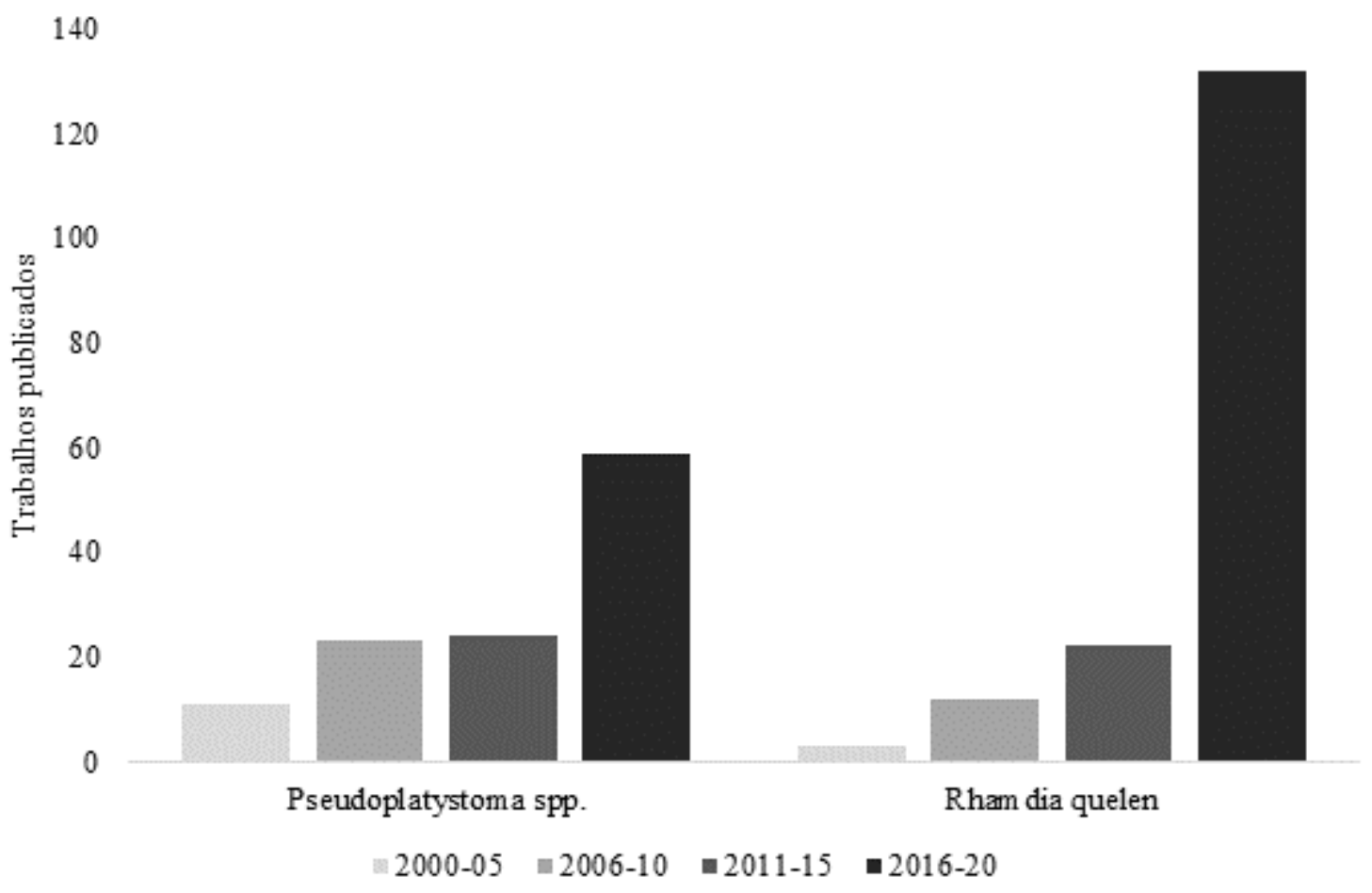

Fonte: Autores.

No entanto, apesar do volume de informação publicada sobre o jundiá, a sua produção ainda é baixa, quando comparado a outras espécies. Neste cenário, emergem algumas perguntas: como são as rações elaboradas especificamente para as espécies de peixes nativos no Brasil? Qual a relação entre os trabalhos já realizados e o que realmente está sendo aplicado nas dietas espécie-específicas em nosso país? Vale ressaltar novamente que as formulações para diferentes espécies são realizadas a partir de literatura existente de espécies exóticas morfo-fisiologicamente similares, principalmente de clima temperado. Este fato pode prejudicar a otimização do desempenho zootécnico, já que existem inúmeras diferenças entre as espécies (hábito alimentar, nível trófico ou de carnivoria, atividade enzimática, sistema digestivo, comportamento, dentre outros) (Baldisserotto \& Gomes, 2013).

\section{Exigência nutricional}

\subsection{Pseudoplatystoma}

As exigências nutricionais desta espécie ainda são pouco conhecidas e há a necessidade de mais estudos acerca da sua nutrição (Matos \& Meurer, 2021). Frequentemente são utilizadas dietas comerciais contendo entre 40 e $42 \%$ de proteína bruta independente da fase de vida dessa espécie (Cunha et al., 2015). Essas formulações são baseadas em exigências nutricionais de peixes carnívoros de água fria, o que levanta questionamentos sobre o real potencial produtivo e zootécnico dessas espécies. Neste sentido, elaborou-se um compilado dos trabalhos mais relevantes relacionados com a nutrição e as principais exigências nutricionais de surubins (Tabela 1).

Pode-se observar uma grande amplitude entre os valores encontrados para as recomendações de proteína (valores entre 28 e 49,25\% PB). Esta variação pode estar relacionada a vários fatores, entre eles: tamanho e genética dos peixes, ingredientes utilizados nas dietas (diferente digestibilidade, palatabilidade e biodisponibilidade de nutrientes), além do balanço de aminoácidos essenciais e da relação energia/proteína (NRC, 2011). Outros fatores como: taxa de alimentação, densidade de 
estocagem, sistema de produção e qualidade da água e do ambiente também podem contribuir significativamente para essas variações nos valores encontrados (Baldisserotto \& Gomes, 2013).

Com relação a exigência em aminoácidos, há um único trabalho (Prado, 2011) que mostra melhorias lineares no desempenho zootécnico conforme a inclusão do aminoácido essencial lisina. No entanto, ele que pode ser inconclusivo, já que o maior nível testado $(2,75 \%)$ pode não ser o ideal de exigência do animal. Campos et al. (2006) avaliaram o perfil de aminoácidos essenciais na carcaça desta espécie, trabalho este que pode servir como modelo para avaliar e estimar a real exigência aminoacídica desses animais.

A exigência de energia para esta espécie é relativamente alta (5140 kcal/kg EB-1 - Martino et al., 2002a) quando comparado a outras espécies como a piracanjuba (3255 kcal $/ \mathrm{kg}$ EB - Borba et al., 2006), o jundiá $(2750 \mathrm{kcal} / \mathrm{kg}$ de ED Bombardelli et al., 2015) e a tilápia (3200 kcal $/ \mathrm{kg}$ de EB - Zead, 2018). Este fato pode estar relacionado a diversos fatores, entre eles o hábito alimentar desta espécie, visto que espécies com hábitos alimentares carnívoros tem uma maior exigência proteica e energética (NRC, 2011).

A relação Energia/Proteína (E/P) influencia na exigência tanto proteica como energética, visto que o excesso de energia na dieta pode limitar o consumo de alimento (NRC, 2011). Quando há uma alta relação E/P, a energia oriunda dos carboidratos e gordura, pode ser suficiente para a realização dos processos fisiológicos. Liberando assim hormônios que indicam a sensação de saciedade, mesmo que ainda não se tenha realizado a ingestão mínima de proteínas e outros nutrientes, essenciais para o crescimento (Baldisserotto, 2013). Se este fato ocorrer, pode haver limitação no crescimento e maior deposição de gordura, diminuindo assim o rendimento de carcaça e a qualidade do produto final (Santos \& Meurer, 2018). No entanto, uma baixa relação E/P também não é interessante, visto que a proteína da dieta pode ser usada como fonte de energia aumentando o custo de produção e a excreção de nitrogênio (NRC, 2011).

Para minerais e vitaminas, há apenas um trabalho publicado com vitamina $\mathrm{C}$, onde foi observada melhoria no crescimento com a adição desta vitamina, além de prevenção contra infestação parasitária e ocorrência de deformidades (Fujimoto \& Carneiro, 2001). A importância da realização de mais trabalhos neste segmento, se fundamenta pelos inúmeros benefícios não somente no desempenho, mas também na saúde e imunidade dos animais suplementados (NRC, 2011).

É notória a importância econômica dos surubins nos últimos anos, principalmente, pelos avanços relacionados à nutrição dessa espécie. No entanto, os estudos ficam divididos entre as espécies e seus híbridos, diluindo o montante das pesquisas. Embora haja essa diluição, ainda fica nítida a variabilidade dos tratamentos na literatura, tornando-os de difícil compilação. Isso se justifica, principalmente, pela presença de nutrientes com valores fixos (ex. proteína e energia), limitando a confiabilidade da recomendação dos autores sobre a relação E/P das dietas. Dessa forma, é necessidade da utilização de metodologias apropriadas (ex. experimentos fatoriais, blocos, entre outros). 
Tabela 1. Resumo dos estudos mais relevantes com surubins (Pseudoplatystoma spp.) relacionados com a nutrição e exigências nutricionais desta espécie.

\begin{tabular}{|c|c|c|c|c|c|c|}
\hline $\begin{array}{c}\text { Parâmetro } \\
\text { testado }\end{array}$ & $\operatorname{Peso}(g)$ & Linhagem & Níveis testados & Recomendado & Dias & Referência \\
\hline \multirow{6}{*}{$\begin{array}{c}\text { Níveis } \\
\text { proteicos }\end{array}$} & 9,69 & Pintachara & 28,32 e $40 \%$ & $\begin{array}{l}40 \% \text { PB até } 75 \text { dias } \\
28 \% \text { PB após } 75 \text { dias }\end{array}$ & 187 & Oliveira et al. (2018) \\
\hline & 8,16 & $P$. reticulatum & $30,35,40,45,50$ e $55 \%$ & $\begin{array}{c}49,25 \% \mathrm{~PB} \text { ou } \\
44,79 \% \mathrm{PD}\end{array}$ & 60 & Cornélio et al. (2014) \\
\hline & 8,4 & Pseudoplatystoma sp & $32,34,36,38$ e $40 \%$ & $40 \% \mathrm{~PB}$ & 70 & Barros et al. (2020) \\
\hline & 13,58 & P. coruscans & 35,40 e $45 \%$ & $40 \% \mathrm{~PB}$ & 50 & Zanardi et al. (2008) \\
\hline & 170 & Pintachara & $27,31,35,39$ e $42 \%$ & 27 a $31 \%$ PD & 40 & Teixeira et al. (2013)* \\
\hline & 356,6 & Pseudoplatystoma sp & 36,38 e $40 \%$ & $38 \% \mathrm{PD}$ & 150 & Honorato et al. $(2015)^{*}$ \\
\hline $\begin{array}{l}\text { Níveis de } \\
\text { lisina }\end{array}$ & 38,2 & Pseudoplatystoma spp. & $1,75,2,00,2,25,2,50$ e $2,75 \%$ & 2,75\% de Lisina & 50 & Prado (2011) \\
\hline \multirow{3}{*}{$\begin{array}{c}\text { Níveis de } \\
\text { energia }\end{array}$} & 2,72 & P. coruscans & $4440,4650,4840$ e $5140 \mathrm{kcal} / \mathrm{kg}$ & $5140 \mathrm{kcal} / \mathrm{kg} \mathrm{EB}$ & 62 & Martino et al. (2002a) \\
\hline & 84,43 & P. reticulatum & $3696,4049,4343,4828$ e $5232 \mathrm{kcal} / \mathrm{kg}$ & $\begin{array}{l}3497 \mathrm{kcal} / \mathrm{kg} \text { ED ou } \\
4238 \mathrm{kcal} / \mathrm{kg} \mathrm{EB}\end{array}$ & 90 & Cunha et al. (2015) \\
\hline & 89,2 & Pintachara & $2865,3105,3345,3585,3825 \mathrm{kcal} / \mathrm{kg}$ & $\begin{array}{l}3345 \text { a } 3585 \mathrm{kcal} / \mathrm{kg} \\
\text { ED }\end{array}$ & 45 & Teixeira et al. (2013) \\
\hline $\begin{array}{c}\text { Fontes } \\
\text { lipídicas }\end{array}$ & 2,75 & P. coruscans & Banha de porco, óleo de milho, soja e linhaça & Não houve diferença & 63 & Martino et al. (2002b) \\
\hline $\begin{array}{c}\text { Relação } \\
\text { Proteína/Lip }\end{array}$ & 1,01 & Pseudoplatystoma sp. & $\begin{array}{c}40,45 \text { e } 50 \% \text { PB } \\
12,16 \text { e } 20 \% \text { de lipídeos }\end{array}$ & $\begin{array}{l}45 \% \text { de PB e } \\
16 \% \text { de Lip }\end{array}$ & 56 & Arslan et al. (2013) \\
\hline $\begin{array}{c}\text { Relação } \\
\text { CHO/Proteína }\end{array}$ & 69,2 & P. reticulatum & $\begin{array}{c}17 \text { e } 30 \% \text { de } \mathrm{CHO} \\
36,40 \text { e } 44 \% \text { de PB } \\
\end{array}$ & $\begin{array}{c}17 \% \text { de } \mathrm{CHO} \text { e } \\
36 \% \text { de } \mathrm{PB} \\
\end{array}$ & 60 & Gonçalves et al. (2018) \\
\hline $\begin{array}{c}\text { Relação } \\
\text { CHO/Lip }\end{array}$ & 5,1 & P. coruscans & $\begin{array}{c}6,5,14,3,14,6,11,4 \text { e } 9,9 \% \text { CHO } \\
19,221,223,0,25,8,27,3 \% \text { Lip }\end{array}$ & $\begin{array}{l}16,5 \mathrm{CHO} \text { e } \\
19,2 \% \mathrm{Lip} \\
\end{array}$ & 64 & Martino et al. (2005) \\
\hline $\begin{array}{c}\text { Substituição } \\
\text { da FP por } \\
\text { GM }\end{array}$ & 113,56 & P. fasciatum & $0,6,12,18,24,30,36$, e $42 \%$ & 10,4 & 49 & Bicudo et al. (2012) \\
\hline Vitamina C & 1,00 & P. corruscans & 0 a $2500 \mathrm{mg} / \mathrm{kg}$ & $>500 \mathrm{mg} / \mathrm{kg}$ & 90 & Fujimoto e Carneiro (2001) \\
\hline
\end{tabular}

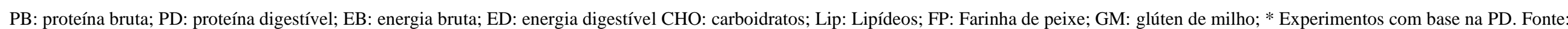
Autores. 


\subsection{Rhamdia quelen}

O jundiá possui suas exigências nutricionais mais bem definidas, se comparado com os peixes do gênero Pseudoplatystoma. Apresenta alguns estudos relacionados à exigência proteica, energética e lipídica, além de apresentar trabalhos voltados a exigências de minerais, carboidratos, aminoácidos e vitaminas (Tabela 2). Os alevinos dessa espécie exibem um crescimento rápido em um curto período e, por esse motivo, apresentam maiores exigências de proteína. Animais mais velhos, por outro lado, apresentam uma taxa de crescimento mais lenta e menor requerimento proteico, já que a necessidade de produção tecidual e/ou de outras estruturas é menor (Santos \& Meurer, 2018). Deste modo, na fase inicial exigem aproximadamente 40\% de PB (Hernández et al., 2018; Signor et al., 2004) e após atingirem em média 25 g, a exigência proteica é reduzida para 35\% de PB (Reidel et al., 2010). Entretanto, recomenda-se a suplementação com lisina e metionina para um maior desempenho, melhora na composição corporal e morfometria intestinal (Schneider et al., 2020). Posteriormente, ao atingirem em média 130g, pode haver uma redução nos valores proteicos para $32 \%$ (Adorian et al., 2017) e já na fase final de crescimento (>350g), dietas com 28 \% de PB são aceitas (Coldebella et al., 2011). Além de níveis proteicos Lazzari et al., (2006) também avaliaram diferentes fontes proteicas. Eles observaram que a combinação entre farelo de soja, farinha de carne e ossos e farinha de peixe apresentou os melhores resultados (Lazzari et al., 2006).

Vale ressaltar que a exigência não deve ser baseada em níveis de proteína, mas sim em um equilíbrio entre os aminoácidos essenciais, já que são absorvidos e direcionados para os tecidos. No caso de suprimento inadequado ou mesmo desequilíbrio aminoacídico, perdas de desempenho dos peixes podem ser ocasionadas (Santos \& Meurer, 2018). A exigência de alguns aminoácidos essenciais já foi determinada, tais como, lisina (Montes-Girao \& Fracalossi, 2006), metionina (Rotili et al., 2018) e triptofano (Pianesso et al., 2015). São trabalhos de grande importância para o avanço nutricional da espécie, no entanto, é necessário que se defina a exigência dos demais aminoácidos para uma formulação mais confiável. 
Tabela 2. Resumo dos estudos mais relevantes com jundiá (Rhamdia quelen) relacionados com a nutrição e exigências nutricionais desta espécie.

\begin{tabular}{|c|c|c|c|c|c|}
\hline Parâmetro testado & Peso (g) & Níveis testados & Nível recomendado & Dias & Referência \\
\hline \multirow{12}{*}{ Níveis proteicos } & 0,3 & $20,27,34$ e $41 \%$ & $40 \%$ & 60 & Melo et al. (2006) \\
\hline & 0,3 & $30,34,38$ e $43 \%$ & $37 \%$ & 30 & Salhi et al. (2004) \\
\hline & 0,78 & $30,34,38,42,46 \%$ & $38 \%$ & 30 & Signor et al. (2004) \\
\hline & 0,78 & 44,51 e $54 \%$ & $51 \%$ & 40 & Piedras et al. (2006) \\
\hline & 1,52 & $26,29,33,37$ e $41 \%(3200 \mathrm{kcal} / \mathrm{kg})$ & $37,3 \%$ & 90 & Meyer e Fracalossi (2004) \\
\hline & 1,52 & $26,29,33,37$ e $41 \%$ (3650 kcal/kg) & $32,6 \%$ & 90 & Meyer e Fracalossi (2004) \\
\hline & 1,86 & 25,30 e $45 \%$ & $45 \%$ & 30 & Hernández et al. (2018) \\
\hline & 26,33 & 38 e $34 \%$ & $34 \%$ & 63 & Schneider et al. (2020) \\
\hline & 47,39 & 25,30 e $35 \%$ & $35 \%$ & 324 & Reidel et al. (2010) \\
\hline & 47,39 & 25,30 e $35 \%$ & $30 \%$ & 324 & Reidel et al. (2021) \\
\hline & 130,05 & 28 e $32 \%$ & $32 \%$ & 90 & Adorian et al. (2017) \\
\hline & 394 & 28,34 e $40 \%$ & $28 \%$ & 90 & Coldebella et al. (2011) \\
\hline Fontes proteicas & 15 & Farinha de carne e ossos, levedura, farelo de soja, farinha de peixe & $\begin{array}{c}\text { Combinação das } \\
\text { farinhas de carne e } \\
\text { ossos e de peixes com } \\
\text { farelo de soja }\end{array}$ & 60 & Lazzari et al. (2006) \\
\hline Lisina & 1,4 & $3,0,4,0,4,5,5,0,5,5,6,0,6,5 \%$ & 4,5 a 5,1 & 119 & Montes-Girao e Fracalossi (2006) \\
\hline Metionina (g/kg) & 3,26 & $3,3,5,98,511,113,8$ e $16,3 \mathrm{~g} / \mathrm{kg}$ & 12,74 a 13,26 & 105 & Rotili et al. (2018) \\
\hline Triptofano (g/kg) & 4,65 & $1,0,1,8,2,6,3,4$ e $4,2 \mathrm{~g} / \mathrm{kg}$ & 2,5 a 3,4 & 60 & Pianesso et al. (2015) \\
\hline Níveis de energia & 44,6 & $2700,2950,3200,3450$ e $3700 \mathrm{kcal}$ & $2700 \mathrm{kcal}$ & 255 & Bombardelli et al. (2015) \\
\hline \multirow{3}{*}{ Fontes lipídicas } & 0,001 & Óleos de canola, girassol e fosfatidilcolina & Óleo de girassol & 28 & Tronco et al. (2007) \\
\hline & 21,58 & Óleo de canola , fígado de bacalhau e banha suína & Óleo de canola & 26 & Melo et al. (2002) \\
\hline & 71,0 & Óleos de arroz, canola ou soja & Óleo de canola & 90 & Losekann et al. (2008) \\
\hline Óleo de Soja & 237,75 & $0,2,4,6,8$ e $10 \%$ & $10 \%$ & 80 & Lazzari et al. (2016) \\
\hline Estrato etéreo & 596 & 8,14 e $20 \%$ & 8 a $14 \%$ & 90 & Coldebella et al. (2013) \\
\hline Relação CHO: Lip & 0,63 & $0,3 / 1$ a $6,4 / 1$ & 5,3: & 75 & Moro et al. (2010) \\
\hline Fibra bruta & 129 & 5 a $9,2 \%$ FB & $9,2 \%$ & 120 & Pedron et al. (2008) \\
\hline Cálcio (g/kg) & 0,10 & 0,8 á 23,9 & $0,8-6,4$ & 30 & Copatti et al. (2017) \\
\hline
\end{tabular}


Research, Society and Development, v. 10, n. 13, e594101321612. 2021

(CC BY 4.0) | ISSN 2525-3409 | DOI: http://dx.doi.org/10.33448/rsd-v10i13.21612

\begin{tabular}{|c|c|c|c|c|c|}
\hline $\begin{array}{c}\text { Propionato de cálcio } \\
\text { e sódio }\end{array}$ & 8,43 & $0,0,25$ e $1 \%$ & $\begin{array}{l}0,25 \% \text { de propionato de } \\
\text { cálcio }\end{array}$ & 60 & Pereira et al. (2018) \\
\hline Selênio & 9,8 & 0,3 e $0,6 \mathrm{mg} / \mathrm{kg}$ de Selênio orgânico e inorgânico & $\begin{array}{c}0,6 \mathrm{mg} / \mathrm{kg} \text { de selênio } \\
\text { orgânico }\end{array}$ & 55 & Piedras et al. (2005) \\
\hline Fósforo & 1,20 & 0,$04 ; 0,30 ; 0,47$ e $0,70 \%$ & $0,53-058$ & 160 & Rocha e Pouey (2020) \\
\hline Vitamina C (mg/kg) & 9,21 & 0 á 1233 & NS & 60 & Borba et al. (2007) \\
\hline Vitamina E (mg/kg) & 55,57 & $0,200,300$ e 400 & 300 & 60 & Uczay et al. (2021) \\
\hline Vitamina A (UI /kg) & 23 & $1500,3000,4500$ e 6000 & 2610 & 70 & Battisti et al. (2017) \\
\hline \multirow{3}{*}{ Hidrolisado proteico } & 11,3 & Sardinha $(0,2,5$ e $10 \%)$ & $10 \%$ & 56 & Ha et al. (2018) \\
\hline & 6,3 & Farelo de soja $(0,5,10,15$ e $20 \%)$ & $20 \%$ & 60 & Uczay et al. (2019b) \\
\hline & 6,42 & Tilápia $(0,5,10,15$ e $20 \%)$ & $20 \%$ & 30 & Uczay et al. (2019a) \\
\hline
\end{tabular}

CHO: Carboidratos; Lip: Lipídeos; FB: Fibra bruta; NS: Não significativo. Fonte: Autores. 
Vale frisar ainda, a importância da relação E/P detalhada anteriormente: quando há uma relação equilibrada pode haver um efeito poupador de proteína. Esse efeito poupador de proteína para o jundiá já foi estudado por Meyer e Fracalossi (2004). Os autores observaram que pode haver uma diminuição de 37,3 \% para 32,6 \% de PB da dieta com um aumento de 3200 para $3650 \mathrm{kcal} / \mathrm{kg}$ de energia. Essa diminuição além de benefícios para o animal, reduzirá os custos da ração e a poluição ambiental (Meyer \& Fracalossi, 2004). Outro trabalho que avaliou a exigência de energia para jundiás foi realizado por Bombardelli et al (2015). Ao utilizarem dietas com 30,5 \% de PD e níveis de energia digestível que variaram entre 2700 e $3700 \mathrm{kcal} / \mathrm{kg}$, esses autores não encontraram diferença para os parâmetros de crescimento. Com isso, concluíram que o menor nível utilizado (2700 kcal/kg), já era suficiente para suprir as exigências energéticas do jundiá com 44,6g (Bombardelli et al., 2015).

Uma das formas mais simples de aumentar o teor de energia da dieta é com a adição de lipídeos, diferentes fontes de lipídeos já foram testadas (Losekann et al., 2008; Melo et al., 2002; Tronco et al., 2007), sendo o óleo de canola e o óleo de girassol os mais recomendados. A relação carboidratos: lipídeos também já foi estudada, sendo que a melhor foi de 5,3: 1 (Moro et al., 2010). Avaliando níveis de óleo de soja na dieta de jundiás, Lazzari e colaboradores (2016) recomendaram a inclusão de $10 \%$. Essa recomendação é devido ao maior desempenho e aumento da deposição ácidos graxos poliinsaturados observado nos filés (Lazzari et al., 2016). Coldebella et al. (2013), por outro lado, mostram efeitos negativos na correta morfometria de ovos e larvas com o uso de até $20 \%$ de lipídeos na dieta. Com isso, o crescimento e sobrevivência das póslarvas foram comprometidos. Os autores concluíram que as melhores respostas reprodutivas foram obtidas com o uso de $8 \mathrm{e}$ 15\% de lipídios nas dietas (Coldebella et al., 2013), corroborando com os resultados de Lazzari et al. (2016).

Os trabalhos com foco na exigência vitamínica-mineral para espécies nativas ainda são escassos. No entanto, eles são necessários visto que a falta ou excesso de minerais e vitaminas podem prejudicar a saúde e o crescimento dos peixes (NRC, 2011). Além de já ser conhecido o fato de haver antagonismo e sinergismo na absorção destes nutrientes, onde a falta ou excesso de um pode limitar a absorção do outro (Elenice et al., 2016). Dentre os poucos trabalhos existentes nesse segmento para o jundiá, estudou-se a suplementação de selênio (Piedras et al., 2005) e cálcio (Copatti et al., 2005) nas dietas desses animais. Observou-se melhor desempenho, sobrevivência e uniformidade do lote de alevinos de jundiá com o uso de 0,6mg/kg de selênio orgânico (Piedras et al., 2005) e melhor crescimento com 0,8 a 6,4g/kg de cálcio (Copatti et al. 2005).

Em se tratando de fontes vitamínicas, trabalhos com o uso de vitamina C (Borba et al., 2007), vitamina E (Uczay et al., 2021) e vitamina A (Battisti et al., 2017) também foram avaliados. Neles, doses entre 0 a $1233 \mathrm{mg} / \mathrm{kg}$ de vitamina $\mathrm{C}$ não afetaram o desempenho (Borba et al., 2007), bem como, a utilização de $300 \mathrm{mg} / \mathrm{kg}$ de vitamina E favoreceu a deposição de ácidos graxos insaturados e melhorou o metabolismo oxidativo dessa espécie (Uczay et al., 2021). A vitamina em níveis de 2610 UI/kg, por sua vez, apresentou melhores resultados de desempenho e menor estresse oxidativo (Battisti et al., 2017).

Embora a quantidade de trabalhos com vitaminas e minerais para os jundiás seja maior do que para o gênero Pseudoplatystoma, esses estudos ainda são escassos e insuficientes para formular uma alimentação adequada e específica para esta espécie. Com isso, as rações produzidas atualmente não são eficientes, o que pode resultar em desempenho insuficiente ou excesso de gastos com alimentação (Santos \& Meurer, 2018).

Recentemente, tem se observado excelentes resultados com a utilização entre 10 e $20 \%$ de hidrolisado proteico em dietas para jundiá (Ha et al., 2019; Uczay et al., 2019b, 2019a). Subprodutos originados a partir da hidrólise enzimática de proteínas presentes em resíduos, que resulta na liberação de pequenos peptídeos e aminoácidos livres mais facilmente absorvidos pelos animais (Uczay et al., 2019a). Essa tecnologia transforma os resíduos em um produto de alto valor biológico e aproveitamento metabólico da fração proteica. Sendo, portanto, uma excelente alternativa para a substituição da farinha de peixe em dietas para jundiá, principalmente na fase de alevinagem. Ainda neste sentido, efeitos positivos no desempenho, composição corporal, melhora nas enzimas digestivas e da atividade antioxidante com efeito prebiótico foram observados (Ha 
et al., 2019; Uczay et al., 2019b). Em outro trabalho com hidrolisados para jundiás foi observado ainda, maior contagem de células caliciformes. Esse aumento indica uma maior proteção, renovação celular e integridade epitelial, ou seja, melhor saúde intestinal dos animais suplementados (Mombach et al., 2019).

Dentre as espécies estudadas nesta revisão, o jundiá é a espécie com as exigências nutricionais mais conhecidas. No entanto, ainda há uma carência de trabalhos com este peixe na fase de crescimento e terminação, obstáculo que pode estar prejudicando a expansão da produção desta espécie.

\section{Considerações Finais}

Nesse artigo de revisão, identificamos quatro pontos relevantes sobre a problemática discutida: (i) necessidade de estratégia nas pesquisas voltadas para os bagres do gênero Pseudoplatystoma, pois os trabalhos publicados variam entre os peixes 'puros' e os híbridos como a Pintachara; recomenda-se que as pesquisas sejam voltadas para essa última espécie, devido suas excelentes características produtivas e tendências no aumento de produção; (ii) é muito importante que haja uma padronização na fabricação das dietas aquícolas, já que em termos produtivos, grande parte das rações comercializadas são na forma extrusada; desta forma, as pesquisas futuras devem manter a confecção de rações extrusadas e não mais na forma peletizada; (iii) são escassas as dietas comerciais específicas para espécies nativas, especialmente em se tratando de micronutrientes, possivelmente impactando a indústria com poucas opções comerciais e linha de produtos; (iv) discrepância entre as pesquisas científicas com nutrição e o volume de produção comercial dessas espécies.

Baseado nesses entraves, o uso de dietas desbalanceadas e inaptas para a criação de peixes nativos, além de não atenderem as reais exigências nutricionais dos animais (por vezes desconhecidas), acarretarão no incremento dos custos produtivos, bem como, do impacto ambiental. Desta forma, deve se reunir esforços para a produção de dietas especificas e de qualidade, principalmente para as fases iniciais, onde as exigências (pelo menos dos macronutrientes) já estão estabelecidas, para que assim tenhamos avanços na produção. Apesar do árduo desafio em desenvolver uma base de dados nutricionais para todas as espécies em questão, algumas espécies de peixes com conhecimento nutricional limitado são criadas comercialmente de forma exitosa. Com isso, há a necessidade de uma estratégia científica, nesse caso na área de nutrição, alinhada com os interesses da indústria, que busque solucionar os maiores desafios enfrentados pela cadeia produtiva (principalmente produtores e empresas de fabricação de ração, no atual momento). Por exemplo: identificar espécies-chave focando estudos científicos na área de desenvolvimento de dietas específicas. Esse diálogo entre os membros da cadeia produtiva e o setor acadêmico é indispensável para a expansão da atividade.

\section{Referências}

Adorian, T. J., Mombach, P. I., Goulart, F. R., Pianesso, D., Fagundes, M. B., Wagner, R., Lazzari, R., Neto, J. R., \& Silva, L. P. da. (2017). Effect of sex and protein level on the intermediary metabolism, growth, deposition of nutrients and profile of volatile compounds of silver catfish (Rhamdia quelen). Aquaculture Nutrition, 1-9. https://doi.org/10.1111/anu.12608

Arslan, M., Dabrowski, K., Ferrer, S., Dietrich, M., \& Rodriguez, G. (2013). Growth, body chemical composition and trypsin activity of South American catfish, surubim (Pseudoplatystoma sp.) juveniles fed different dietary protein and lipid levels. Aquaculture Research, 44(5), 760-771. https://doi.org/10.1111/j.1365-2109.2011.03081.x

Baldisserotto, B. (2013). Fisiologia de peixes aplicada à piscicultura (3a ed.).

Baldisserotto, B., \& Gomes, L. de C. (2013). Espécies nativas para piscicultura no Brasil. (2a ed.).

Barros, R. P., Luz, J. R., Ramos, A. P. de S., Costa, D. S., \& Braga, L. G. T. (2020). Exigência em proteína bruta do híbrido jundiara (Pseudoplatystoma fasciatum x Leiarius marmoratus). Research, Society and Development, 9(8), 1-17. https://doi.org/10.33448/rsd-v9i8.6866

Battisti, E. K., Marasca, S., Durigon, E. G., Villes, V. S., Schneider, T. L. S., Uczay, J., Peixoto, N. C., \& Lazzari, R. (2017). Growth and oxidative parameters of Rhamdia quelen fed dietary levels of vitamin A. Aquaculture, 474, 11-17. https://doi.org/http://dx.doi.org/10.1016/j.aquaculture.2017.03.025 
Bibiano Melo, J. F., Lundstedt, L. M., Metón, I., Baanante, I. V., \& Moraes, G. (2006). Effects of dietary levels of protein on nitrogenous metabolism of Rhamdia quelen (Teleostei: Pimelodidae). Comparative Biochemistry and Physiology - A Molecular and Integrative Physiology, 145(2), 181-187. https://doi.org/10.1016/j.cbpa.2006.06.007

Bicudo, Á. J. de A., Borghesi, R., Dairiki, J. K., Sado, R. Y., \& Cyrino, J. E. P. (2012). Performance of juveniles of Pseudoplatystoma fasciatum fed graded levels of corn gluten meal. Pesquisa Agropecuaria Brasileira, 47(6), 838-845. https://doi.org/10.1590/S0100-204X2012000600015

Bombardelli, R. A., Sanches, E. A., Tessaro, L., Buzzi, A. H., Martins, C. V. B., \& Meurer, F. (2015). Digestible energy requirement for females of Rhamdia quelen on reproductive activity fed with ration based on vegetal ingredients. Latin American Journal of Aquatic Research, 43(3), 566-574. https://doi.org/10.3856/vol43-issue3-fulltext-18

Borba, M. R., Fracalossi, D. M., \& Freitas, F. A. de. (2007). Efeito da suplementação de vitamina C na dieta sobre a susceptibilidade de alevinos de jundiá, Rhamdia quelen, ao Ichthyophthirius multifiliis. Acta Scientiarum - Animal Sciences, 29(1), 93-99.

Borba, M. R., Fracalossi, D. M., \& Pezzato, L. E. (2006). Dietary energy requirement of piracanjuba fingerlings, Brycon orbignyanus, and relative utilization of dietary carbohydrate and lipid. Aquaculture Nutrition, 12(3), 183-191. https://doi.org/10.1111/j.1365-2095.2006.00401.x

Broggi, J. A., Wosniak, B., Uczay, J., Pessatti, M. L., \& Fabregat, T. E. H. P. (2017). Hidrolisado proteico de resíduo de sardinha como atrativo alimentar para juvenis de jundiá? Arquivo Brasileiro de Medicina Veterinaria e Zootecnia, 69(2), 505-512. https://doi.org/10.1590/1678-4162-8348

Campos, P., Martino, R. C., \& Trugo, L. C. (2006). Amino acid composition of Brazilian surubim fish (Pseudoplatystoma coruscans) fed diets with different levels and sources of fat. Food Chemistry, 96(1), 126-130. https://doi.org/10.1016/j.foodchem.2005.02.017

Coldebella, I. J., Emanuelli, T., Veiverberg, C. A., Pretto, A., Rossato, S., Ferreira, D., Barcellos, L. J. G., \& Neto, J. R. (2013). Effect of different dietary lipid levels on the reproduction of Rhamdia quelen (Quoy and Gaimard, 1824). Aquaculture Nutrition, 19(5), 751-764. https://doi.org/10.1111/anu.12022

Coldebella, I. J., Neto, J. R., Mallmann, C. A., Veiverberg, C. A., Bergamin, G. T., Pedron, F. A., Ferreira, D., \& Barcellos, L. J. G. (2011). The effects of different protein levels in the diet on reproductive indexes of Rhamdia quelen females. Aquaculture, 312(1-4), 137-144. https://doi.org/10.1016/j.aquaculture.2010.12.021

Copatti, C. E., Coldebella, I. J., Raduz-Neto, J., Garcia, L. O., Rocha, M. C. Da, \& Baldisserotto, B. (2017). Effect of dietary calcium on growth and survival of silver catfish fingerlings, Rhamdia quelen (Heptapteridae), exposed to different water pH. Aquaculture Nutrition, 11, 345-350. https://doi.org/10.1111/j.1365-2095.2005.00355.x

Cornélio, F. H. G., Cunha, D. A. da, \& Silveira, J. (2014). Dietary protein requirement of juvenile cachara catfish, Pseudoplatystoma reticulatum. Journal of the World Aquaculture Society, 45(1), 45-54. https://doi.org/10.1111/jwas.12090

Crepaldi, D. V, Faria, P. M. C., Teixeira, E. D. A., Ribeiro, L. P., P, Â. A., Melo, D. C. De, Cintra, A. P. R., Prado, S. D. A., Costa, F. A. A., Drumond, M. L., Lopes, V. E., \& Moraes, V. E. De. (2007). Biologia reprodutiva do surubim (Pseudoplatystoma coruscans). Revista Brasileira de Reprodução Animal, 30(3), $159-167$.

Cunha, D. A. Da, Cornélio, F. H. G., \& Fracalossi, D. M. (2015). Exigência de energia em dietas para juvenis de cachara, Pseudoplatystoma reticulatum. Boletim Do Instituto de Pesca, 41(3), 567-578.

Cyrino, J. E. P., De Almeida Bicudo, Á. J., Sado, R. Y., Borghesi, R., \& Dairiki, J. K. (2010). A piscicultura e o ambiente - o uso de alimentos ambientalmente corretos em piscicultura. Revista Brasileira de Zootecnia, 39(SUPPL. 1), 68-87. https://doi.org/10.1590/S1516-35982010001300009

De Seixas Filho, J. T., Brás, J. D. M., Gomide, A. T. D. M., Oliveira, M. G. D. A., Donzele, J. L., \& Menin, E. (2001). Anatomia Funcional e Morfometria do Intestino no Teleostei (Pisces) de Água Doce Surubim (Pseudoplatystoma coruscans - Agassiz, 1829). Revista Brasileira de Zootecnia, 30(6), 1670-1680. https://doi.org/10.1590/s1516-35982001000700003

Elenice, S. dos R. G., Aldi, F., Wilson, R. eacute rio B., Marcio, D. G., \& Altevir, S. (2016). Supplementation of selenium and vitamin E in diets for pacu (Piaractus mesopotamicus): Effect on performance, body yields and lipid stability. African Journal of Agricultural Research, 11(2), 65-73. https://doi.org/10.5897/ajar2015.10296

Fracalossi, D. M., Meyer, G., Santamaria, F. M., Weingartner, M., \& Zaniboni Filho, E. (2004). Desempenho do jundiá, Rhamdia quelen, e do dourado, Salminus brasiliensis, em viveiros de terra na região sul do Brasil. Acta Scientiarum. Animal Sciences, 26(3), 345-352. https://doi.org/10.4025/actascianimsci.v26i3.1806

Fujimoto, R. Y., \& Carneiro, D. J. (2001). Adição de ascorbil polifosfato, como fonte de vitamina C, em dietas para alevinos de pintado, Pseudoplatystoma corruscans (Agassiz , 1829). Acta Scientiarum, 23(4), 855-861.

Gonçalves, A. F. N. (2014). Desempenho produtivo e respostas fisiológicas de juvenis de cachara (Pseudoplatystoma reticulatum) alimentados com diferentes níveis de proteína e carboidrato. 50.

Gonçalves, A. F. N., Ha, N., Biller-Takahashi, J. D., Gimbo, R. Y., Urbinati, E. C., \& Takahashi, L. S. (2018). Dietary protein-to-carbohydrate ratios affect metabolism and growth of juvenile surubim cachara (Pseudoplatystoma reticulatum). Aquaculture International, 26(1), 349-362. https://doi.org/10.1007/s10499-017-0213-3

Ha, N., Jesus, G. F. A., Gonçalves, A. F. N., de Oliveira, N. S., Sugai, J. K., Pessatti, M. L., Mouriño, J. L. P., \& El Hadi Perez Fabregat, T. (2019). Sardine (Sardinella spp.) protein hydrolysate as growth promoter in South American catfish (Rhamdia quelen) feeding: Productive performance, digestive enzymes activity, morphometry and intestinal microbiology. Aquaculture, 500, 99-106. https://doi.org/10.1016/j.aquaculture.2018.10.004

Hemre, G.-I., Mommsen, T. P., \& Krogdahl, A. (2002). Carbohydrates in fish nutrition: effects on growth, glucose metabolism and hepatic enzymes. Aquaculture Nutrition, 8, 175-194. 
Hernández, D. R., Barrios, C. E., Santinón, J. J., Sánchez, S., \& Baldisserotto, B. (2018). Effect of fasting and feeding on growth, intestinal morphology and enteroendocrine cell density in Rhamdia quelen juveniles. Aquaculture Research, 49(4), 1512-1520. https://doi.org/10.1111/are.13606

Honorato, C. A., Ushizima, T. T., Santamaria, F. M., Flores-Quintana, C. I., Marcondes, V. M., \& Nascimento, C. A. (2015). Desempenho produtivo e econômica de surubins (Pseudoplatystoma sp.) alimentados com níveis de proteína e estocados em tanque-rede. Arquivo Brasileiro de Medicina Veterinaria e Zootecnia, 67(5), 1408-1414. https://doi.org/10.1590/1678-4162-7238

IBGE. (2017). Produção da Pecuária Municipal 2017.

Junior, H. A., Garcia, S., \& Mello, G. L. de. (2011). Jundiá: Um peixe sob medida para a Região Sul do Brasil. Panorama Da Aquicultura, 2 , 2011.

Lazzari, R., Emanuelli, T., Maschio, D., Ferreira, C. C., Battisti, E. K., \& Radünz-Neto, J. (2016). The inclusion of soybean oil in the diets of silver catfish (Rhamdia quelen) in relation to growth quality and fillet acceptability. Latin American Journal of Aquatic Research, 44, 39-45. https://doi.org/10.3856/vol44issue 5-fulltext-4

Lazzari, R., Neto, J. R., Emanuelli, T., Pedron, F. de A., Costa, M. L., Losekann, M. E., Correia, V., \& Bochi, V. C. (2006). Diferentes fontes protéicas para a alimentação do jundiá (Rhamdia quelen). Ciência Rural, 36(1), 240-246.

Losekann, M. E., Neto, J. R., Emanuelli, T., De Araújo Pedron, F., Lazzari, R., Bergamin, G. T., Corrêia, V., \& Simões, R. S. (2008). Alimentação do jundiá com dietas contendo óleos de arroz, canola ou soja. Ciencia Rural, 38(1), 225-230. https://doi.org/10.1590/S0103-84782008000100036

Martino, R. C., Cyrino, J. E. P., Portz, L., \& Trugo, L. C. (2005). Performance, carcass composition and nutrient utilization of surubim Pseudoplatystoma coruscans (Agassiz) fed diets with varying carbohydrate and lipid levels. Aquaculture Nutrition, 11(2), 131-137. https://doi.org/10.1111/j.13652095.2004.00332.x

Martino, Ricardo C., Cyrino, J. E. P., Portz, L., \& Trugo, L. C. (2002a). Effect of dietary lipid level on nutritional performance of the surubim, Pseudoplatystoma coruscans. Aquaculture, 209(1-4), 209-218. https://doi.org/10.1016/S0044-8486(01)00738-4

Martino, Ricardo C., Cyrino, J. E. P., Portz, L., \& Trugo, L. C. (2002b). Performance and fatty acid composition of surubim (Pseudoplatystoma coruscans) fed diets with animal and plant lipids. Aquaculture, 209, 233-246. https://doi.org/S0044-8486(01)00847-X

Matos, E. J. A., \& Meurer, F. (2021). Nutrition of Surubim (Pseudoplatystoma spp) and its hybrids - literature review. Brazilian Journal of Animal and Environmental Research, 4(2), 2320-2335. https://doi.org/10.34188/bjaerv4n2-062

Melo, J. F. B., Neto, J. R., Silva, J. H. S. da, \& Trombetta, C. G. (2002). Desenvolvimento e composição corporal de alevinos de jundiá (Rhamdia Quelen) alimentados com dietas contendo diferentes fontes de lipídios. Ciência Rural, 33(2), 323-327.

Meyer, G., \& Fracalossi, D. M. (2004). Protein requirement of jundia fingerlings, Rhamdia quelen, at two dietary energy concentrations. Aquaculture, 240(14), 331-343. https://doi.org/10.1016/j.aquaculture.2004.01.034

Mombach, P. I., Adorian, T. J., Pianesso, D., Santos, T. S. dos, Lima, J. S., Robalo, S. S., \& Leila, P. (2019). Pectic hydrolysates in the diet of silver catfish (Rhamdia quelen): Growth performance, blood and liver biochemistry, histological parameters and intestinal contents. Aquaculture Nutrition, November 2018, 1-10. https://doi.org/10.1111/anu.12958

Montes-Girao, P. J., \& Fracalossi, D. M. (2006). Dietary lysine requirement as basis to estimate the essential dietary amino acid profile for Jundiá, Rhamdia quelen. Journal of the World Aquaculture Society, 37(4), 388-396.

Moro, G. V., Camilo, R. Y., Moraes, G., \& Fracalossi, D. M. (2010). Dietary non-protein energy sources: Growth, digestive enzyme activities and nutrient utilization by the catfish jundiá, Rhamdia quelen. Aquaculture Research, 41(3), 394-400. https://doi.org/10.1111/j.1365-2109.2009.02352.x

NRC. (2011). National Research Council. Nutrient Requirements of Fish and Shrimp. National Academies Press.

Oliveira, C. M., Piñeyro, J. I. G., \& Sousa, R. G. C. (2018). Análise zootécnica do Pseudoplatystoma spp. (Pintachara) submetido a teores de proteínas distintos. Biota Amazónia, 8(4), 17-20. https://doi.org/10.18561/2179-5746/biotaamazonia.v8n4p17-20

Parra, J. E. G., Radünz Neto, J., Veiverberg, C. A., Lazzari, R., Bergamin, G. T., Pedron, F. D. A., Rossato, S., \& Sutili, F. J. (2008). Alimentação de fêmeas de jundiá com fontes lipídicas e sua relação com o desenvolvimento embrionário e larval. Ciencia Rural, 38(7), 2011-2017. https://doi.org/10.1590/S010384782008000700033

Pedron, F. D. A., Neto, J. R., Emanuelli, T., Da Silva, L. P., Lazzari, R., Corrêia, V., Bergamin, G. T., \& Veiverberg, C. A. (2008). Cultivo de jundiás alimentados com dietas com casca de soja ou de algodão. Pesquisa Agropecuaria Brasileira, 43(1), 93-98. https://doi.org/10.1590/S0100$204 X 2008000100012$

Peixe BR. (2021). Peixe BR 2021. In Anuário 2021 Peixe BR da Piscicultura.

Pereira, S. A., Oliveira, H. M., Jesus, G. F. A., Addam, K. G. S., Silva, B. C., Yamashita, M. M., Lehmann, N. B., Martins, M. L., \& Mouriño, J. L. P. (2018). Can the minerals calcium and sodium, chelated to propionic acid, influence the health and zootechnical parameters of native silver catfish Rhamdia quelen? Aquaculture, 496, 88-95. https://doi.org/10.1016/j.aquaculture.2018.07.016

Pianesso, D., Radünz Neto, J., da Silva, L. P., Goulart, F. R., Adorian, T. J., Mombach, P. I., Loureiro, B. B., Dalcin, M. O., Rotili, D. A., \& Lazzari, R. (2015). Determination of tryptophan requirements for juvenile silver catfish (Rhamdia quelen) and its effects on growth performance, plasma and hepatic metabolites and digestive enzymes activity. Animal Feed Science and Technology, 210, 172-183. https://doi.org/10.1016/j.anifeedsci.2015.09.025

Piedras, S. R. N., Morae, P. R. R., Isoldi, L. A., Pouey, J. L. O. F., \& Rutz, F. (2005). Comparação entre o selênio orgânico e o inorgânico empregados na dieta de alevinos de jundiá (Rhamdia quelen). Boletim Do Instituto de Pesca, 31(2), 171-174. 
Piedras, S. R. N., Pouey, J. L. O. F., Moraes, P. R. R., \& Rodrigues, F. V. (2006). Resposta de alevinos de jundiá (Rhamdia sp.) Alimentados com diferentes níveis de proteína bruta e energia digestível. Revista Brasileira de Agrociência, 12(2), 217-220.

Prado, S. de A. (2011). Exigência de lisina de juvenis de surubim (Pseudoplatystoma spp). Universidade Federal De Minas Gerais Escola De Veterinária.

Reidel, A., Boscolo, W. R., Feiden, A., \& Romagosa, E. (2010). The effect of diets with different levels of protein and energy on the process of final maturation of the gametes of Rhamdia quelen stocked in cages. Aquaculture, 298(3-4), 354-359. https://doi.org/10.1016/j.aquaculture.2009.11.005

Reidel, A., Signor, A. A., Feiden, A., Boscolo, W. R., \& Romagoza, E. (2021). Crescimento do jundiá (Rhamdia quelen) criado em tanques-rede com diferentes níveis de proteína e energia na dieta. Research, Society and Development, 10(2), 1-12. https://doi.org/10.33448/rsd-v10i2.12638

Rocha, C. B., \& Pouey, J. L. O. F. (2020). Exigência de fósforo para juvenis de jundiá (Rhamdia quelen) em uma dieta semipurificada. Research, Society and Development, 9(8), 1-15. https://doi.org/10.33448/rsd-v9i8.5842

Rotili, D. A., Rossato, S., Freitas, I. L., Martinelli, S. G., Neto, J. R., \& Lazzari, R. (2017). Determination of methionine requirement of juvenile silver catfish (Rhamdia quelen) and its effects on growth performance, plasma and hepatic metabolites at a constant cystine level. Aquaculture Research, 1-9. https://doi.org/10.1111/are.13530

Salhi, M., Bessonart, M., \& Chediak, G. (2004). Growth, feed utilization and body composition of black catfish, Rhamdia quelen, fry fed diets containing different protein and energy levels. Aquaculture, 231, 435-444. https://doi.org/10.1016/j.aquaculture.2003.08.006

Santos, H. K. dos, \& Meurer, F. (2018). Nutrition and feeding aspects for Jundiá (Rhamdia quelen) Rhamdia quelen nutrition and feeding. Reviews in Aquaculture, 1-11. https://doi.org/10.1111/raq.12318

Schneider, T. L. S., Durigon, E. G., Peixoto, N. C., Uczay, J., Molinari, M., \& Lazzari, R. (2020). Effects of reduced protein level and dietary amino acid supplementation on growth, body composition and intestinal morphometry of silver catfish (Rhamdia quelen). Aquaculture Research, 51(12), 4925-4937. https://doi.org/10.1111/are.14830

Signor, A., Signor, A. A., Feiden, A., Boscolo, W. R., Reidel, A., \& Hayashi, C. (2004). Exigência de proteína bruta para alevinos de jundiá Rhamdia quelen. Revista Varria Scientia, 04(08), 79-89.

Teixeira, E. A., Euler, A. C. C., Faria, P. M. C., Turra, E. M., Luz, R. K., Prado, S. A., Takata, R., Ribeiro, P. A. P., Fontes, D. O., Ribeiro, L. P., \& Saliba, E. O. S. (2013). Performance and nutrient utilization in South American juvenile catfish Pseudoplatystoma spp. weighting $89-170 \mathrm{~g}$, fed at different energy and protein levels. Arqivo Brasileiro de Medicina Veterinária e Zootecnia, 65, 1500-1508.

Tronco, A. P., Radünz Neto, J., Medeiros, T. D. S., \& Lima, R. L. De. (2007). Alimentação de larvas de jundiá (Rhamdia quelen) com dietas semipurificadas e fontes lipídicas. Boletim Do Instituto de Pesca, 33(1), 9-17.

Uczay, J., Battisti, E. K., Lazzari, R., Pessatti, M. L., Schneider, T. L., Hermes, L. B., Peixoto, N. C., \& Fabregat, T. E. H. P. (2019a). Tilapia protein hydrolyzate improves growth performance, protein absorption and antioxidant status in Silver catfish (Rhamdia quelen). Aquaculture Research, 50(11), 31923201. https://doi.org/10.1111/are.14273

Uczay, J., Battisti, E. K., Lazzari, R., Pessatti, M. L., Schneider, T. L. S., Hermes, L. B., Peixoto, N. C., \& Fabregat, T. E. H. P. (2019b). Fish meal replaced by hydrolysed soybean meal in diets increases growth and improves the antioxidant defense system of silver catfish (Rhamdia quelen). Aquaculture Research, 50(5), 1438-1447. https://doi.org/10.1111/are.14019

Uczay, J., Saccol, E. M. H., Pês, T. S., Durigon, E. G., Peixoto, N. C., Pavanato, M. A., Baldisserotto, B., Emanuelli, T., \& Lazzari, R. (2021). Tocopherol in silver catfish diets reduces oxidative stress and improves the unsaturated fatty acid profile. Aquaculture Research, 52(6), 2818-2827. https://doi.org/10.1111/are.15133

Valenti, W. C., Barros, H. P., Moraes-Valenti, P., Bueno, G. W., \& Cavalli, R. O. (2021). Aquaculture in Brazil: past, present and future. Aquaculture Reports, 19(July 2020), 100611. https://doi.org/10.1016/j.aqrep.2021.100611

Villares-Junior, G. A., \& Goitein, R. (2015). Differences in the feeding of Rhamdia quelen (Siluriformes , Heptapteridae) in four distinct lotic systems. Brazilian Journal of Biology, 75(3), 650-654.

Zanardi, M. F., Boquembuzo, J. E., \& Koberstein, T. C. R. D. (2008). Desempenho de juvenis de pintado (Pseudoplatystoma coruscans) alimentados com três diferentes dietas. Rev. Acad., Ciênc. Agrár. Ambient., 6(4), 445-450.

Zead, M. Y. A. (2018). Effect of different protein and energy levels on growth performance and body composition of red tilapia. Animal Biotechnology, April, 81-88. 